\title{
Heterogeneous reorganization of actin filaments in living endothelial cells in response to shear stress
}

\author{
Toshihiro Sera ${ }^{1, *}$, Marie Terada ${ }^{2}$, Susumu Kudo ${ }^{1}$ \\ ${ }^{I}$ Department of Mechanical Engineering, Faculty of Engineering, Kyushu University \\ ${ }^{2}$ Division of Mechanical Engineering, Graduate School of Engineering and Science, Shibaura Institute of Technology
}

Received: 16 March, 2020 / Accepted: 27 May, 2020

(C) Japanese Society of Biorheology 2020

\begin{abstract}
In this study, we investigated the spatial and temporal reorganization of actin filaments in living endothelial cells in response to shear stress by transfecting a fluorescent protein, Dronpa-Green-labeled actin, which was photoactivated microscopically by UV irradiation, and evaluating the time constants of fluorescence decay after photoactivation. The time constant in the upstream region decreased gradually after $30 \mathrm{~min}$ of shear stress and then increased. Particularly, the time constant in the downstream region tended to be higher than in the upstream region, suggesting that actin polymerization was more activated in the downstream region. Our results demonstrated the spatial and temporal heterogeneity of actin reorganization due to shear stress.
\end{abstract}

Keywords actin reorganization, Dronpa-Green, shear stress, photoactivation

\section{Introduction}

Vascular endothelial cells (ECs) are constantly exposed to various mechanical stimuli, such as fluid shear stress and vasodilation/contraction, and their cytoskeleton is deeply involved in mechanotransduction signaling [1]. Cytoskeletal polymers transmit shear forces to the membrane attachment sites, where conformational changes in associated proteins initiate signaling, and structural changes in the cytoskeletal connections alter the properties of the transmitted force [1-3]. Actin filaments are the most abundant cytoskeletal polymers. These filaments are located in close proximity to cell membranes and play an important role in transmitting mechanical stimuli to the cell. Indeed, increased actin filament turnover precedes a decrease in the amount of

*E-mail: sera@mech.kyushu-u.ac.jp polymerized actin, revealing that cytoskeletal depolymerization characterizes the early phase of the endothelial shear stress response [3].

The actin cytoskeleton is linked to integrins, transmembrane receptors for extracellular matrix (ECM) proteins at focal adhesions (FAs), via several cytoplasmic proteins, including vinculin and talin, and is anchored to the ECM. As a result, the linkage between integrin and the actin cytoskeleton at FAs is exposed to mechanical forces through the ECMs. FAs are dynamic structures in which cellular morphological changes can be modulated by the regulation of localization or levels of specific cytoplasmic proteins [4]. Mechanical stretching can induce an accumulation of actin filaments at an integrin cluster, and these actin filaments are involved in strengthening the linkage [5]. Actin polymerization at peripheral FAs has been induced by mechanical stretching, even when the motor protein myosin II activity was inhibited [6]. Moreover, the accumulation of zyxin as an FA protein depends on mechanical stretching, indicating that zyxin is involved in the mechanical-force-dependent facilitation of actin polymerization at FAs [6].

The EC shape and cytoskeletal structure aligned parallel to the direction of shear stress in regions of unidirectional flow [2, 7-9]. Shear stress induced the spatiotemporal distribution of mechanical strain in the intermediate filament cytoskeleton near the basal surface, resulting in changes in the conformation or organization of molecules within the FA sites [10] and, particularly, shear-stress-induced FA dynamic alignment [11]. Additionally, the spatially coordinated changes in the structural dynamics of the cytoskeleton and FAs agreed with the focusing of mechanical stress and/ or strain near major sites of shear-stress-mediated mechanotransduction [12]. During the first 6 min after flow onset, the flow-induced actin remodeling in the subconfluent bovine aortic EC (BAEC) layer was more prominent in the 
downstream and side directions than in the upstream direction [13]. These results suggested that the reorganization of actin filaments may exhibit heterogeneity in the cytosol in response to shear stress.

In this study, we investigated the spatial and temporal reorganization of actin filaments in response to shear stress. However, immunostaining did not allow the time-lapse imaging of the same cell. Fluorescent proteins, such as GFP, have been used to observe actin during live cell imaging, but they were not suitable for observing the spatial and temporal responses of a specific region as the whole cell was fluorescent. Thus, to achieve our objective, we constructed a fluorescent protein, Dronpa-Green labeled actin, which was photoactivated microscopically by UV irradiation [14].

\section{Materials and Methods}

\section{cDNA construction}

Hybrid cDNA encoding the fusion of human cytoplasmic $\beta$-actin (AcGFP1-actin, Plasmid 632453; Clontech, Mountain View, CA, USA) and Dronpa-Green (DG1; DG1-MN1, AM-V0073M; Medical \& Biological Laboratories Co., Ltd., Nagoya, Japan) [14] were inserted in multiple cloning sites on a pcDNATM3.1(+) (V790-20; Invitrogen, Carlsbad, CA, USA) mammalian expression vector. We designed the linker that connected the actin to DG1 on the basis of the AcGFP1-actin. The cDNA encoding actin and DG1 were amplified by polymerase chain reaction (PCR) using the following primers: 5'-actin, 5'-CAGACTCGAGCTATG GATGATGATATCGCC-3' and 3'-actin, 5'-TCTAGACTC GAGCCTAGAAGCATTT-3'; 5'-DG, 5'-GCCGCGGGA TCCATGGTGAGTGTGATTAAACCAGAC-3' and 3'-DG, 5'-GAATTCAGCTCGAGATCTGAGTCCGGACTTGGC CTGCCTCGG-3'. The digested DG and actin fragments were ligated to the digested vector, generating the DG1actin fusion construct.

\section{Cell culture and transfection}

BAECs (Toyobo Co., Ltd., Osaka, Japan) were cultured in Dulbecco's modified Eagle's medium (DMEM; Gibco ${ }^{\mathrm{TM}}$, Thermo Fisher Scientific, Waltham, MA, USA) containing 1\% antibiotic-antimycotic $(10,000 \mathrm{U} / \mathrm{mL}$ penicillin, $10,000 \mu \mathrm{g} / \mathrm{mL}$ streptomycin, and $25 \mu \mathrm{g} / \mathrm{mL}$ amphotericin B; Gibco ${ }^{\mathrm{TM}}$, Thermo Fisher Scientific) and 10\% (v/v) fetal bovine serum (Biological Industries, Kibbutz Beit HaEmek, Israel) in humidified incubators with $5 \% \mathrm{CO}_{2}$ at $37^{\circ} \mathrm{C}$.

Prior to transfection, BAECs were seeded in $35 \mathrm{~mm}$ glass-bottomed dishes and cultured to $70 \%$ confluence. BAECs adhering to the glass-bottomed dishes were washed twice with Opti-MEM (Gibco ${ }^{\mathrm{TM}}$, Thermo Fisher Scientific). We exchanged the DMEM with $1 \mathrm{~mL}$ of Opti-MEM, and the BAECs were cultured for $14-16 \mathrm{~h}$. Then, $2 \mu \mathrm{L}$ of DNA
$(1 \mu \mathrm{g} / \mu \mathrm{L})$ in $100 \mu \mathrm{L}$ of Opti-MEM was mixed with $4 \mu \mathrm{L}$ of HilyMax (Dojindo Laboratories, Kumamoto, Japan). This solution, including the construct DNA, was then added to the BAECs adhering to the glass-bottomed dish with $1 \mathrm{~mL}$ of Opti-MEM. Then, DG1-actin or DG1-MN was transfected into the BAECs. We used BAECs (Passages 6-10) grown to confluence for experiments two days after transfection.

Before exposure of shear stress to the BAECs, to confirm the characteristics of actin in DG1-actin, $0.5 \mu \mathrm{M}$ Cytochalasin D (CytoD; Enzo Life Sciences, Farmingdale, NY, USA) was diluted in the flow medium to induce actin disassembly.

\section{Experimental device and imaging}

A conventional parallel-plate-type flow chamber was used to apply shear stress to the BAECs. The shear stress applied to the cells $(\tau=2 \mathrm{~Pa})$ is expressed using the following equation $[15]$ :

$$
\tau=6 Q \mu /\left(w h^{2}\right)
$$

where $Q$ is the flow rate, $\mu$ is the viscosity of the medium $\left(8.4 \times 10^{-4} \mathrm{P} \cdot \mathrm{s}\right), w$ is the channel width $(14 \mathrm{~mm})$, and $h$ is the channel height $(0.27 \mathrm{~mm})$. The flow through the chamber was approximated as two-dimensional fully developed laminar flow. The flow chamber was set into the flow circuit filled with medium; the chamber was placed between the upper and lower reservoirs to achieve a steady flow, and the temperature in the circuit was maintained at $37^{\circ} \mathrm{C}$.

The chamber was placed on the stage of an inverted microscope (Eclipse TE2000-S; Nikon Corp., Tokyo, Japan) with a $40 \times$ oil immersion lens (S-Fluo, NA $=1.30$; Nikon Corp.). The microscope stage was maintained at $37^{\circ} \mathrm{C}$ by enclosing it in thermal insulation material. To estimate the actin filament's response to shear stress, we focused on the fluorescence decay due to the diffusion of DG1-actin in the photoactivated region. In this study, a cell was divided into two regions based on the nucleus and flow direction; upstream and downstream regions, and the upstream region was defined as the side faced to the flow. Conversely, the downstream region was defined as the other side of the upstream region. After photobleaching the cytoplasm by strong irradiation using a $488 \mathrm{~nm}$ laser for $10 \mathrm{~s}$, DG1-actin was photoactivated by UV irradiation at a specific region with a $2.5 \mu \mathrm{m}$ diameter (20 Hz, Polaris II; New Wave Research) every 10 min for $60 \mathrm{~min}$ or every $60 \mathrm{~min}$ for $180 \mathrm{~min}$. Immediately after photoactivation, fluorescent images were acquired every $0.22 \mathrm{~s}$ for $120 \mathrm{~s}$ using weak irradiation with a $488 \mathrm{~nm}$ laser. In the light path, a $500 \mathrm{~nm}$ dichroic mirror and a 540 $\pm 5 \mathrm{~nm}$ emission filter were used. The excitation light was emitted by a xenon lamp (C7773; Hamamatsu Photonics K.K., Hamamatsu, Japan), and the wavelength was filtered using an AquaCosmos system (C7501; Hamamatsu Photonics K.K.). The fluorescence emissions from the sample were amplified using an image intensifier (C8600-03; Hamamatsu 
Photonics K.K.), detected using a charge-coupled device camera (C6790; Hamamatsu Photonics K.K.), and recorded on a personal computer. The fluorescence images were then analyzed using ImageJ [16], and the decay of the relative fluorescence intensity observed after photoactivation was fitted by the following exponential decay function:

$$
\frac{F(t)}{F_{0}}=e^{-\frac{1}{\tau^{*}} t},
$$

where $F_{0}$ and $F(t)$ are the fluorescence intensities at time $t=$ 0 and $t$ after UV irradiation, respectively, and $\tau^{*}$ is a time constant. In this study, we calculated $\tau^{*}$ to achieve $t=\tau^{*}$.

\section{Statistical analysis}

All statistical analyses were performed using $\mathrm{R}$ version 3.5.0 [17] . Multiple comparisons were performed using analysis of variance and Tukey's test. Differences were considered significant when $p<0.05$. The number of glassbottomed culture dishes and that of cells are denoted by $N$ and $n$, respectively.

\section{Results}

We first characterized the expression and localization of DG1-actin in BAECs using colony PCR. The insert length of DG1-actin was approximately 2,000 bp, and DG1-actin was detected in all of the colonies (Fig. 1A). Next, we observed BAECs transfected with DG1-actin, AcGFP-actin, and DG1-MN1. The distributions of DG1-actin and AcGFPactin were stronger in the cytoplasm than in the nucleus (Figs. 1B and 1C). In contrast, the distribution of DG1MN1 was homogenous (Fig. 1D). We also confirmed the fluorescence recovery of DG1-actin by UV irradiation. Fluorescence was observed in BAECs transfected with DG1-actin and DG1-MN1 treated by UV irradiation, as indicated by the arrowheads in Figs. 1E-1H. In addition, in BAECs not treated with CytoD, the fluorescence around the cell membranes remained relatively constant during a 40 min shear stress (Figs. 2A1-2A5). In contrast, in BAECs treated with CytoD, the fluorescence around the membranes decreased gradually during the shear stress (Figs. 2B1-2B5).

Figure $3 \mathrm{~A}$ shows the representative decay for the normalized fluorescent intensity $\left(F(t) / F_{0}\right)$ in upstream and downstream region after $30 \mathrm{~min}$ of shear stress, and the rate of fluorescence decay was slightly slower in downstream region than in upstream region. Figure 3B shows the heterogeneous reorganization of actin in response to shear stress for $60 \mathrm{~min}$. No statistically significant differences were found between the upstream and downstream regions. However, after 10 min of shear stress, $\tau^{*}$ in the upstream and downstream regions was $93.4 \pm 11.7 \mathrm{~s}$ and $78.4 \pm 5.2 \mathrm{~s}$ (mean \pm standard error [SE]), respectively. The actin reorganization in the upstream region was particularly biphasic; hence, $\tau^{*}$ in the upstream region tended to decrease. The $\tau^{*}$ value was lowest at $74.6 \pm 4.9 \mathrm{~s}$ after $30 \mathrm{~min}$ of shear stress, and then it increased and the $\tau^{*}$ value was $86.4 \pm 9.9 \mathrm{~s}$ after $60 \mathrm{~min}$ of shear stress. In contrast, after $30 \mathrm{~min}$ of shear stress, $\tau^{*}$ was higher in the downstream region than in the upstream region and the $\tau^{*}$ value was $83.1 \pm 5.0 \mathrm{~s}$, indicating that actin disassembly in response to shear stress was activated in the upstream region more than in the downstream region (Fig. 3A). At $60 \mathrm{~min}, \tau^{*}$ in both regions was similar. Figure $3 \mathrm{C}$ shows the heterogeneous response of actin to shear stress between 60 and $180 \mathrm{~min}$. In the upstream region, $\tau^{*}$ remained relatively constant at approximately $95 \mathrm{~s}$. However, it gradually increased in the downstream region to $133.2 \pm 16.1 \mathrm{~s}$ after $180 \mathrm{~min}$ of shear stress, indicating that actin polymerization in response to shear stress might have been promoted in the downstream region.

\section{Discussion}

In this study, the spatial and temporal responses to shear stress by actin filaments in BAECs using DG1-actin as a fusion protein of actin and fluorescent protein were examined. We evaluated $\tau^{*}$ as the ratio of fluorescence decay after photoactivation [18]. The fluorescence decay rate of photoactivated DG1-actin reflected actin monomer reorganization in the cytoplasm, and low and high $\tau^{*}$ indicated actin disassembly and polymerization, respectively [18]. Regarding the spatial response, we focused on the upstream and downstream regions of the flow and found that the shear stress induced heterogeneous actin responses in BAECs. Particularly, $\tau^{*}$ in the upstream region decreased gradually with 30 min of shear stress and then increased. In addition, $\tau^{*}$ was smaller in the upstream region than in the downstream region after 30,40 , and $180 \mathrm{~min}$, indicating that actin reorganization in the cell was not homogeneous and that disassembly was activated in the upstream region more than in the downstream region.

We used DG1-actin to examine the response of actin to shear stress. When BAECs transfected with DG1-actin were irradiated by a weak excitation light of $488 \mathrm{~nm}$, the cells fluoresced without the nucleus (Fig. 1B). BAECs were photobleached by a strong excitation light at $488 \mathrm{~nm}$ and then photoactivated again using UV irradiation (Figs. 1F and $1 \mathrm{H})$. These results indicated that DG1-actin retained the characteristics of DG1. In addition, to confirm that DG1-actin exhibited the characteristics of actin, BAECs transfected with DG1-actin were treated by CytoD as an inhibitor of actin disassembly. In cells treated with CytoD, the fluorescence around the cell membranes decreased over time, whereas no decrease was observed in fluorescence near the cell membranes of the cells that were not treated with CytoD (Fig. 2). This suggested that actin polymerization was inhibited by CytoD and that DG1-actin exhibited the characteristics of intact actin. 

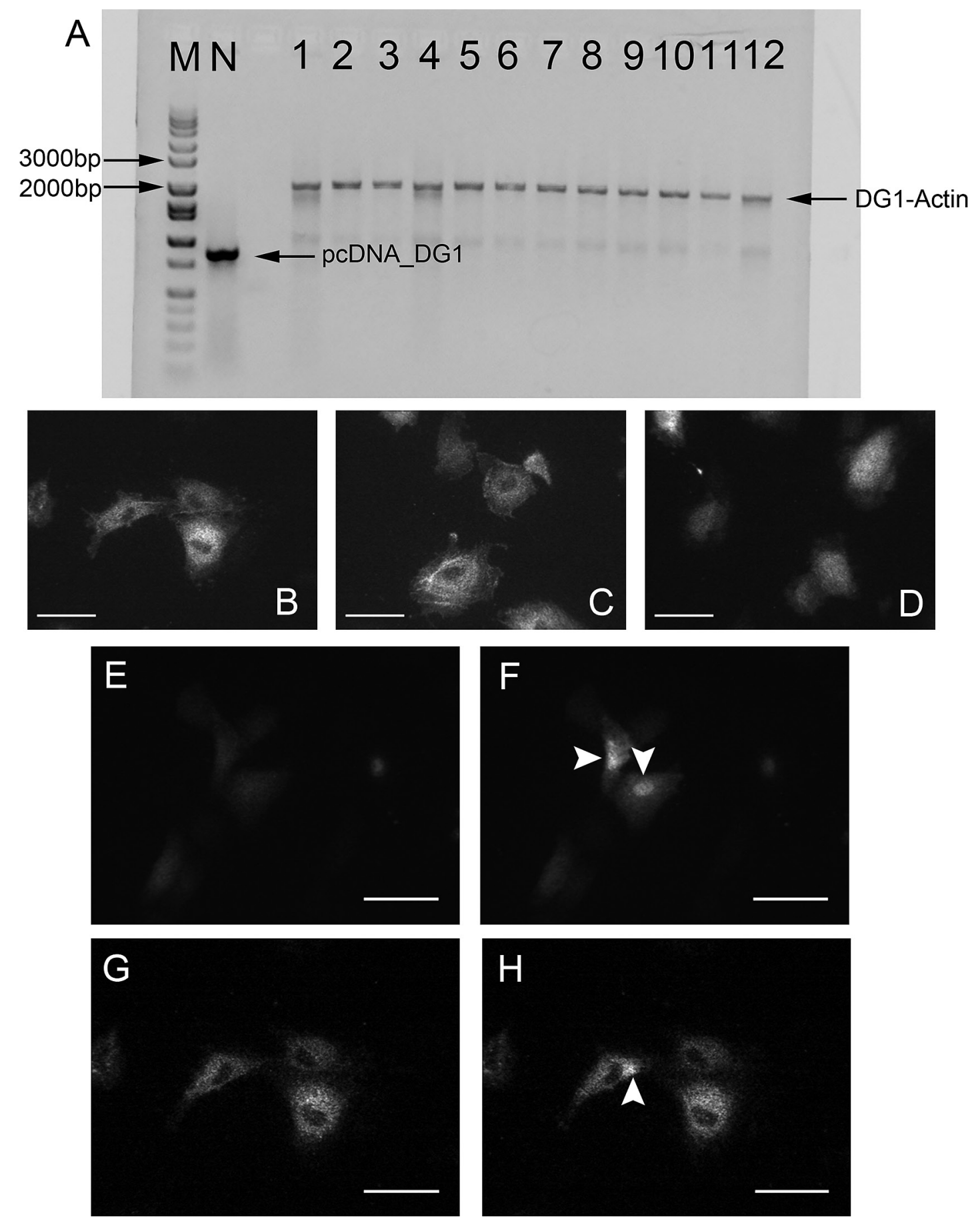

Fig. 1 Characterization of DG1-actin expressed in BAECs via colony PCR, fluorescence microcopy, and photoactivation by UV irradiation. (A) Colony PCR of pcDNA_DG1 and DG1-actin. (B) Representative BAECs transfected with DG1-actin. (C) Representative BAECs transfected with AcGFP-actin. (D) Representative BAECs transfected with DG1-MN. Characteristics of DG1-MN (E, F) and DG1-actin (G, H). Panels (E) and $(\mathrm{G})$ show representative images after photobleaching by strong irradiation using a $488 \mathrm{~nm}$ laser for $10 \mathrm{~s}$. Panels $(\mathrm{F})$ and $(\mathrm{H})$ show representative images after photoactivation by UV irradiation. The arrowheads indicate the photoactivated region. Scale bar: $30 \mu \mathrm{m}$.

Our study demonstrated the heterogeneous reorganization of actin in response to shear stress. In particular, actin disassembly in response to shear stress tended to be activated in the upstream region more than in the downstream region at 30, 40, and $180 \mathrm{~min}$ (Fig. 3). Shear stress modulated the cell structure and various functions, including actin organization. In a previous study, a $3 \mathrm{~Pa}$ shear stress for $180 \mathrm{~min}$ induced reorganization concomitant with the disappearance of the dense peripheral bands of microfilaments and the formation of stress fibers aligned with the long axes of elongating cells [4]. Additionally, an upstream concentration of vinculin was observed, which might play a 

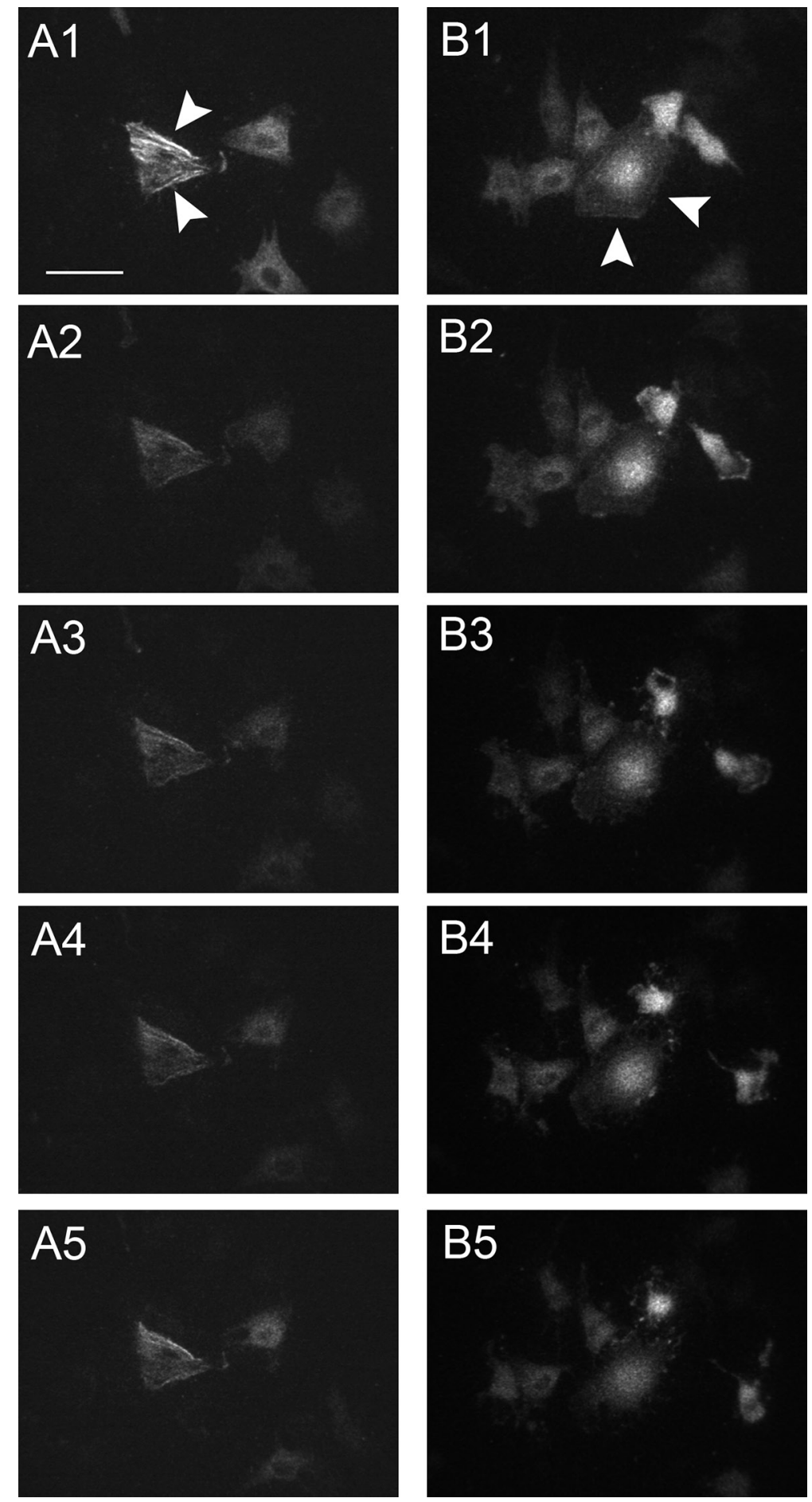

Fig. 2 Response of actin to shear stress in (A1-A5) BAECs and (B1-B5) BAECs treated with $0.5 \mu \mathrm{M}$ CytoD. (A1, B1) Before shear stress, (A2, B2) $10 \mathrm{~min}$ load, (A3, B3) $20 \mathrm{~min}$ load, (A4, B4) $30 \mathrm{~min}$ load, and (A5, B5) $40 \mathrm{~min}$ load. The actin indicated by arrowheads in (A1) remained during a 40 min shear stress; however, the actin indicated by arrowheads in (B1) was disassembled. Scale bar: $30 \mu \mathrm{m}$.

role in anchoring stress fibers, specifically at the upstream ends of the cells [4]. The results of this study indicated that actin in the upstream region might be reorganized as a result of shear stress after $180 \mathrm{~min}$ and that actin in the downstream region might conversely be stabilized, which is consistent with our results. However, our results showed that actin disassembly in the upstream region was activated during the initial response to shear stress (at 30 and $40 \mathrm{~min}$ ) more than in the downstream region, even though previous studies did not show preferential alignment or orientation of newly formed stressed fibers $[4,19]$. When confluent MDCK cells were exposed to a shear stress of $0.074 \mathrm{~Pa}$, 

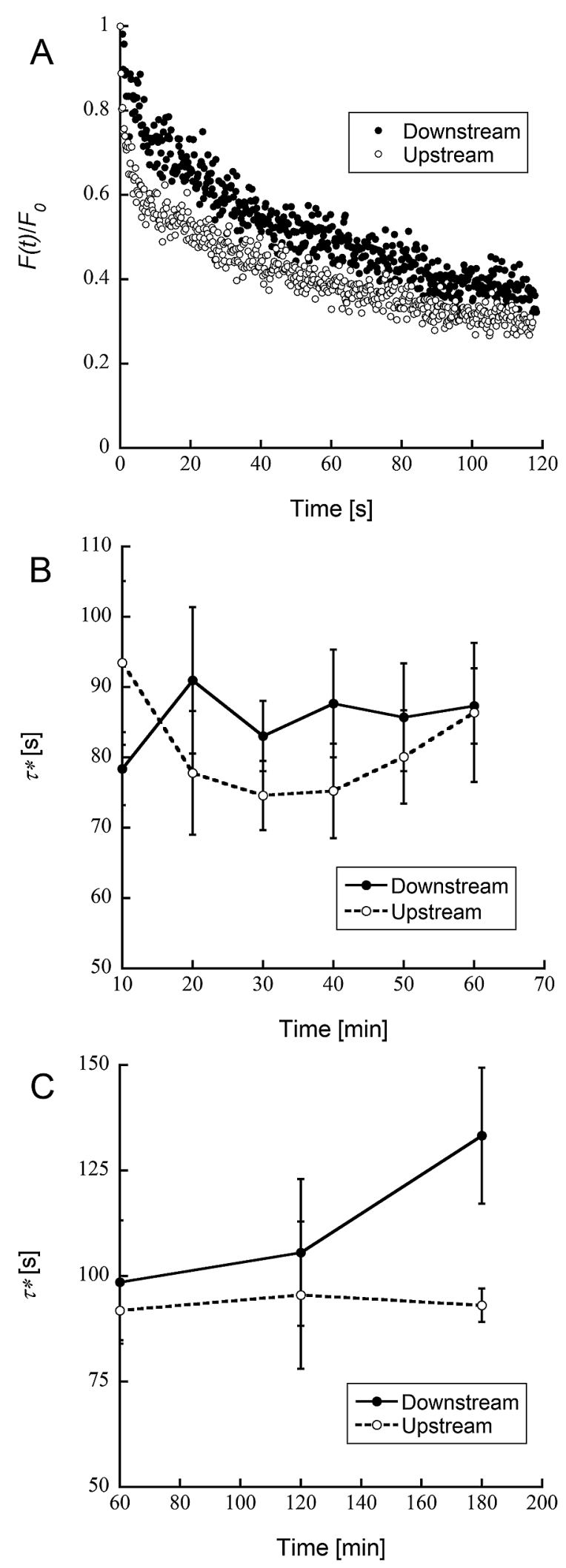

Fig. 3 The representative decay for the normalized fluorescent intensity $\left(F(t) / F_{0}\right)$ in upstream and downstream region after 30 min of shear stress (A). $F_{0}$ and $F(t)$ are the fluorescence intensities at time $t=0$ and $t$ after UV irradiation, respectively. Time constant of the relative fluorescence intensity decay after photoactivation in the upstream and downstream regions during $60 \min (\mathrm{B}, N=8, n=10)$ and $180 \min (\mathrm{C}$, $N=3, n=4)$ shear stresses. Values in $\mathrm{B} \& \mathrm{C}$ are represented as means $\pm \mathrm{SE}$. which was similar to the physiological shear stress of renal epithelial cells, FAs in the interior region, mainly in the upstream region, diminished after $40 \mathrm{~min}$ of shear stress, which was associated with the disassembly of linked actin stress fibers [20]. Small GTPase Rho has been implicated in the mediation of cellular responses to mechanical stimuli and has been shown to exhibit biphasic activity during shear stress [21]. A shear stress of 1.2 Pa subjected to BAECs triggered a decrease in Rho activity after 5-15 min, followed by an increase that peaked at $60 \mathrm{~min}$; the initial phase of the low Rho activity was associated with a decrease in stress fibers, whereas the later restoration of Rho activity corresponded to an increase in stress fibers [21]. This increase and decrease in stress fibers may be related to the assembly and disassembly of actin, respectively. However, this previous study did not focus on the heterogeneity of Rho activity. Our results suggested biphasic activity of actin in response to shear stress, particularly in the upstream region.

In summary, we investigated the spatial and temporal reorganization of actin filaments in BAECs in response to shear stress by evaluating the fluorescence decay of photoactivated DG1-actin. The time constant of the fluorescence decay was higher in the downstream region than in the upstream region at 30,40 , and $180 \mathrm{~min}$, indicating that actin polymerization was activated in the downstream region more than in the upstream region. Our results suggested that actin might exhibit spatial and temporal heterogeneous responses to shear stress.

\section{References}

1. Helmke BP, Davies PF. The cytoskeleton under external fluid mechanical forces: hemodynamic forces acting on the endothelium. Ann Biomed Eng. 2002; 30: 284-96.

2. Davies PF, Zilberberg J, Helmke BP. Spatial microstimuli in endothelial mechanosignaling. Circ Res. 2003; 92: 359-70. doi:10.1161/01.RES.0000060201.41923.88.

3. Osborn EA, Rabodzey A, Dewey CF, Hartwig JH. Endothelial actin cytoskeleton remodeling during mechanostimulation with fluid shear stress. Am J Physiol Cell Physiol. 2006; 290: C44452. doi:10.1152/ajpcell.00218.2005.

4. Girard PR, Nerem RM. Shear stress modulates endothelial cell morphology and F-actin organization through the regulation of focal adhesion-associated proteins. J Cell Physiol. 1995; 163: 179-93. doi:10.1002/jcp.1041630121.

5. Glogauer M, Arora P, Yao G, Sokholov I, Ferrier J, McCulloch CA. Calcium ions and tyrosine phosphorylation interact coordinately with actin to regulate cytoprotective responses to stretching. J Cell Sci. 1997; 110(Pt 1): 11-21.

6. Hirata H, Tatsumi H, Sokabe M. Mechanical forces facilitate actin polymerization at focal adhesions in a zyxin-dependent manner. $\mathrm{J}$ Cell Sci. 2008; 121: 2795-804. doi:10.1242/jcs.030320.

7. Dewey CF, Bussolari SR, Gimbrone MA, Davies PF. The dynamic response of vascular endothelial cells to fluid shear stress. J Biomech Eng. 1981; 103: 177-85.

8. Malek AM, Izumo S. Mechanism of endothelial cell shape change and cytoskeletal remodeling in response to fluid shear stress. J Cell Sci. 1996; 109(Pt 4): 713-26. 
9. Galbraith CG, Skalak R, Chien S. Shear stress induces spatial reorganization of the endothelial cell cytoskeleton. Cell Motil Cytoskeleton. 1998; 40: 317-30. doi:10.1002/(SICI)10970169(1998)40:4<317::AID-CM1>3.0.CO;2-8.

10. Helmke BP, Rosen AB, Davies PF. Mapping mechanical strain of an endogenous cytoskeletal network in living endothelial cells. Biophys J. 2003; 84: 2691-9. doi:10.1016/S0006-3495(03)75074-7.

11. Davies PF, Robotewskyj A, Griem ML. Quantitative studies of endothelial cell adhesion. Directional remodeling of focal adhesion sites in response to flow forces. J Clin Invest. 1994; 93: 2031-8. doi:10.1172/JCI117197.

12. Mott RE, Helmke BP. Mapping the dynamics of shear stressinduced structural changes in endothelial cells. Am J Physiol Cell Physiol. 2007; 293: C1616-26. doi:10.1152/ajpcell.00457.2006.

13. Choi CK, Helmke BP. Short-term shear stress induces rapid actin dynamics in living endothelial cells. Mol Cell Biomech MCB. 2008; 5: 247-58.

14. Ando R, Mizuno H, Miyawaki A. Regulated fast nucleocytoplasmic shuttling observed by reversible protein highlighting. Science. 2004; 306: 1370-3. doi:10.1126/science.1102506.

15. Franco D, Milde F, Klingauf M, Orsenigo F, Dejana E, Poulikakos $\mathrm{D}$, et al. Accelerated endothelial wound healing on microstructured substrates under flow. Biomaterials. 2013; 34: 1488-97. doi:10.1016/ j.biomaterials.2012.10.007.

16. Schneider CA, Rasband WS, Eliceiri KW. NIH Image to ImageJ: 25 years of image analysis. Nat Methods. 2012; 9: 671-5.

17. R Core Team. R: a language and environment for statistical computing. R Found Stat Comput. 2013: 16.

18. Kiuchi T, Ohashi K, Kurita S, Mizuno K. Cofilin promotes stimulusinduced lamellipodium formation by generating an abundant supply of actin monomers. J Cell Biol. 2007; 177: 465-76. doi:10.1083/jcb.200610005.

19. Franke RP, Gräfe M, Schnittler H, Seiffge D, Mittermayer C, Drenckhahn D. Induction of human vascular endothelial stress fibres by fluid shear stress. Nature. 1984; 307: 648-9.

20. Verma D, Meng F, Sachs F, Hua SZ. Flow-induced focal adhesion remodeling mediated by local cytoskeletal stresses and reorganization. Cell Adhes Migr. 2015; 9: 432-40. doi:10.1080/19336918. 2015.1089379.

21. Tzima E, del Pozo MA, Shattil SJ, Chien S, Schwartz MA. Activation of integrins in endothelial cells by fluid shear stress mediates Rho-dependent cytoskeletal alignment. EMBO J. 2001; 20: 4639-47. doi:10.1093/emboj/20.17.4639. 